\title{
The JAK-STAT Pathway and the JAK Inhibitors
}

\author{
Ana Paula Galli Sanchez ${ }^{1}$, Tatiane Ester Aidar Fernandes ${ }^{2}$, and Gustavo Martelli Palomino ${ }^{3}$ \\ ${ }^{1}$ Dermatologist and Master of Science from the Medical College of the University of São Paulo, Medical Contributor to Severe Psoriasis Clinic \\ of the Complexo Hospitalar Padre Bento de Guarulhos, Brazil \\ ${ }^{2}$ Dermatologist, São Paulo, Brazil \\ ${ }^{3}$ Biologist, Master and PhD from the Program of Basic and Applied Immunology, School of Medicine of Ribeirão Preto, University of São \\ Paulo, Ribeirão Preto, Brazil
}

\begin{abstract}
Received: November 17, 2020; Accepted: November 26, 2020; Published: November 30, 2020
*Corresponding author: Ana Paula Galli Sanchez, Dermatologist and Master of Science from the Medical College of the University of São Paulo, Medical Contributor to Severe Psoriasis Clinic of the Complexo Hospitalar Padre Bento de Guarulhos, Brazil. E-mail: apc-med@hotmail.com
\end{abstract}

\section{Abstract}

Dozens of cytokines that bind Type I and Type II receptors use the Janus Kinases (JAK) and the Signal Transducer and Activator of Transcription (STAT) proteins pathway for intracellular signaling, orchestrating hematopoiesis, inducing inflammation, and controlling the immune response. Currently, oral JAK inhibitors are being used to treat many inflammatory and myeloproliferative diseases and are also under investigation in several clinical trials for skin diseases. Thus, dermatologists should understand how the JAK-STAT pathway works as well as the mechanism of action of the JAK inhibitors which will certainly become an important part of the dermatologist's treatment armamentarium in the next few years.
\end{abstract}

Keywords: JAK inhibitors; Janus Kinases; JAK-STAT Pathway

\section{List of Abbreviations:}

AD: Atopic Dermatitis

ADP: Adenosine diphosphate

Dmards: Disease-Modifying Antirheumatic Drugs

JAK: Janus kinase(s)

Jaki: Janus kinase Inhibitor(s)

PIAS: Protein Inhibitor of Activated STAT

P-STAT: Phosphorylated STAT

STAT: Signal Transducer and Activator of Transcription

TYK2: Tyrosine Kinase 2

Wsxws: Tryptophan-Serine-X-Tryptophan-Serine

\section{Introduction}

Cytokines are small proteins responsible for intercellular communication in the immune system and beyond. They are structurally and functionally heterogeneous, binding and signaling through ligand-specific receptors on target cells. Receptor engagement activates intracellular signaling pathways that elicit target cell responses [1-3,13].

Cytokine-target therapies are being employed as part of the dermatology clinical practice for some years, targeting cytokines outside the cells. More recently, small-molecule drugs such as the oral Janus kinase inhibitors (JAKi), which target tyrosine kinases involved in intracellular signaling, are being used to treat myeloproliferative neoplasms, rheumatological, and inflammatory bowel diseases and are also under investigation in several clinical trials for dermatological conditions (e.g. atopic dermatitis (AD), psoriasis, alopecia areata, vitiligo, systemic sclerosis, actinic dermatitis, sarcoidosis, palmoplantar pustulosis, and amyopathic dermatomyositis-associated lung disease) [1,411].

In the near future, JAKi will become an important part of the dermatologist's treatment armamentarium [7-10,12]. Thus, dermatologists should understand how the JAKi function. This article not only reviews the Janus Kinase (JAK) - Signal Transducer and Activator of Transcription (STAT) protein signaling (JAKSTAT pathway) but also the mechanism of action of the first and second-generation JAKi.

The Janus kinases (JAK1, JAK2, JAK3, and Tyrosine kinase 2 TYK2) are intracellular tyrosine kinases that catalyze the transfer 
of $\mathrm{y}$-phosphate from Adenosine Triphosphate (ATP) to protein tyrosine residues, having an essential role in the signaling of numerous cytokines [1-3]. The STATs comprise an important class of seven molecules (STAT1, STA2, STAT3, STAT4, STAT5A, STAT5B, and STAT6) that transmit signals from cytokine receptors to the cell nucleus and drive gene transcription $[1,2,6,10]$.

\section{The JAK-STAT pathway}

The JAK-STAT pathway signaling is important for relaying messages of multiple cytokines from outside the cell to its inside $[1-3,6,10,13]$.

Cytokines can be classified based on its membership in families: the tumor necrosis factors family; the interleukins family; the interferons family; the transforming growth factors beta family, and the chemokines family. Cytokines of the interferons family and most of the interleukins family (except the members of the IL-1 and IL-17 families) bind Type I and Type II receptors which activate JAKs to initiate the signal transduction [1-3,6,10-14].

More than 50 cytokines, hormones, and growth factors use the JAK-STAT pathway for intracellular signaling [4-6,11,14,15]. Cytokines that signal via the JAK-STAT pathway are helical bundle cytokines and exhibit broad pleiotropic actions, orchestrating hematopoiesis, inducing inflammation, and controlling the immune response [1-5,9-11,14,15].

Type I and II receptors are quite similar and consist of multiple (usually two) protein chains which oligomerize upon binding of the ligand in order to initiate intracellular signaling $[1,2,11,14]$. One chain binds the cytokine with high-affinity, and the other chain binds a lower-affinity site on the cytokine [14]. Each functional receptor complex has two receptor chains with long intracellular regions where there are docking sites for the JAKs and the STATs $[1,14]$.

Type I cytokine receptor family contains a conserved extracellular WSXWS (tryptophan-serine-X-tryptophan-serine, where $\mathrm{X}$ is any amino acid) motif and is made up of four subfamilies: the common gamma $(\gamma c)$ family (used by IL-2 family cytokines), the common beta $(\beta c)$ family (used by IL-3 family cytokines), the gp130 family (used by IL-6 family cytokines), and the growth factor and hormones receptors family $[1,2,11,14,16]$. Besides, IL-4 and IL-13 (which share IL-4R $\alpha$ ) and IL-12 and IL-23 (which share IL-12R $\beta 1$ ) also utilize Type I cytokine receptors. All these "class I cytokines" consist of four $\alpha$-helices and employ Type I receptors[11,14].

Type II receptors do not have the WSXWS motif and recognize interferons and IL-10 family cytokines, i.e., "class II cytokines", which are five or six helices proteins $[1,2,14]$.

In mammals, the four JAKs are constitutively yet non-covalently associated with the intracellular domains of Type I/II receptor chains $[1,2,6,10,14]$.

JAK1, JAK2, and TYK2 are expressed indiscriminately throughout the body, whereas JAK3 is expressed in the hematopoietic cells. JAK1 acts by transmitting the signals of numerous proinflammatory cytokines, JAK 2 regulates erythropoiesis, myelopoiesis, and inflammation and the main function of both JAK3 and TYK2 is to regulate the immune system $[2,3,14,15]$.

Upon cytokine binding to the extracellular portion of its specific receptor chain, dimerization (or higher order complexes) of receptor subunits occurs, bringing two JAKs close to one another. Therefore, the activation of the JAK-STAT signaling pathway initiates through the recruitment and trans-phosphorylation of a pair of distinct JAKs associated with the intracellular receptor chains (Figure 1) $[1,3,14]$. JAK2 is the exception, as it is the only JAK which could pair with itself. JAK2 is associated with the receptors of cytokines (IL-3, IL-5, INF-y), hormones (GH, prolactin, leptin), and growth factors (granulocyte macrophage colony-stimulating factor, erythropoietin) [2-4,14]. Thus, JAK2 regulates functions including erythropoiesis, myelopoiesis, and inflammation [1-4,14].

Some Type I and II receptor subunits associate selectively with a specific JAK, whereas others are less selective [4,14]. For instance, gp130 chains can use JAK1, JAK2, and possibly TYK2. JAK3 is mainly expressed in various hematopoietic tissue cells $[3,14]$ and specifically associates with yc chains $[4,14]$, which pair exclusively with JAK1-associated receptor chains. JAK1 pairs with the other three JAKs enzyme except with itself [3] being essential for signaling by all class II cytokine receptors, yc using cytokines, and gp130 using cytokines [4]. TYK2 regulates phosphorylation of STAT proteins downstream of the receptors for cytokines IL-12 and IL-23 (where it pairs with JAK2) as well as Type I interferons and IL-10 family cytokines (where it pairs with JAK1) [3].

As mentioned, different kinds of cytokine binding triggers receptor dimerization, which leads to JAK enzymes activation, i.e. the two JAKs phosphorylate each other (trans-phosphorylation) $[3,4,10,11,14]$. Activated JAKs then phosphorylate tyrosine residues within the cytokine receptor intracellular domains, creating docking sites for the recruitment of the STATs in the phosphorylated receptor chains 1-4, 11, 14. STATs bind active, phosphorylated receptors and are, in turn, phosphorylated by the JAKs. Phosphorylated STAT (p-STAT) proteins form homodimers, heterodimers or tetramers which translocate into the nucleus where they induce the transcription of target genes (Figure 1) [1$4,6,11,14]$.

Each cytokine receptor complex recruits and employs a specific combination of JAKs, having the propensity to activate a particular STAT (Table 1) $[10,11,14]$. For example, in the yc family of cytokines, IL-4 dominantly activates STAT6. On the other hand, IL-2, IL-7, IL-9, and IL-15 primarily activate STAT5A and STAT5B (and to some degree activate STAT3 and STAT1) [16]. Therefore, different cytokine receptors bind different combinations of JAKs to activate different programs in cells $[2,11,14]$.

The JAK-STAT signaling pathway is switched off by suppressor proteins which act to attenuate cytokine signaling at multiple levels $[6,11,14,17,18]$. One key regulatory module involves a list of phosphatases, which hydrolyze phosphorylated tyrosine residues on JAKs, STATs, and upstream receptors [6]. Suppressor of Cytokine Signaling (SOCS) proteins are inhibitors of the JAKSTAT pathways that bind to JAKs or cytokine receptors, thereby 


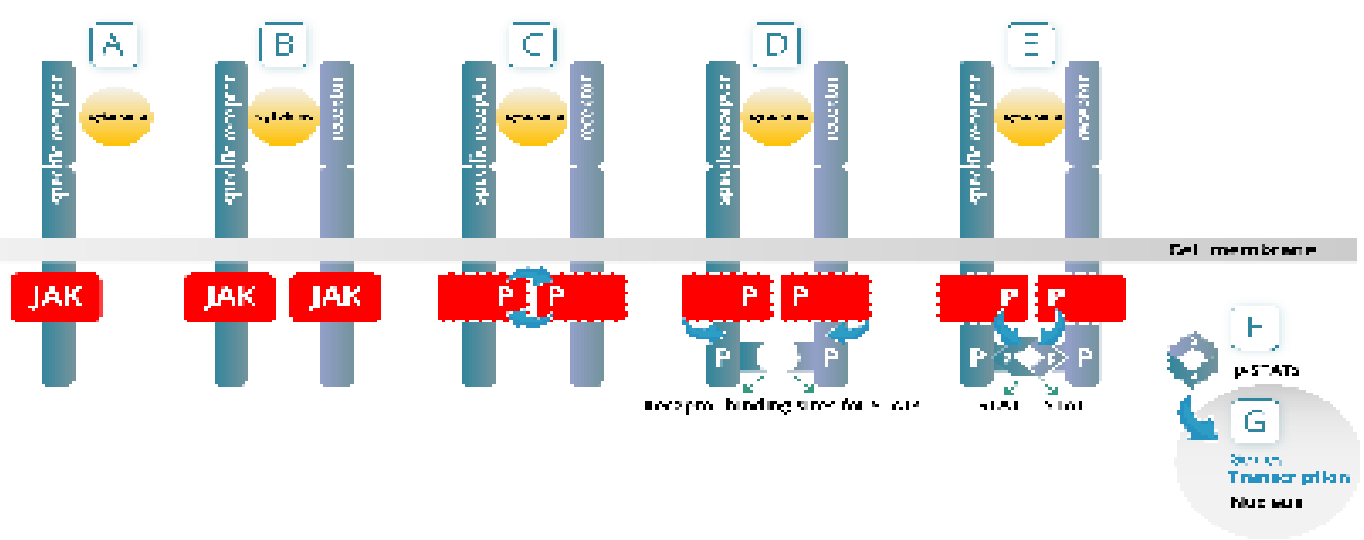

Figure 1: (A) Cytokine binds to its specific receptor on the cell membrane. (B) Specific cytokine receptor subunit dimerizes with another receptor subunit which brings the JAKs associated with each receptor subunit proximal. (C) The phosphorylation (P) of JAKs occurs (activation of JAKs). (D) Activated JAKs then phosphorylate tyrosine residues within the cytokine receptor intracellular domains, creating binging sites for the STATs proteins. (E) STATs proteins bind active, phosphorylated receptors and are, in turn, phosphorylated by JAKs. (F) p-STAT proteins form homo- and heteropolymers which translocate into the nucleus. (G) p-STAT proteins induce transcription of target genes.

Table 1: Summary of ligands, associated Janus Kinase (JAK), Signal Transduction and Activator of transcription (STAT) molecules.

\begin{tabular}{|c|c|c|c|}
\hline & Ligand/cytokine & Associated JAK & Associated STAT \\
\hline \multirow[t]{21}{*}{ Type I cytokine receptors } & IL-2 & JAK1, JAK3 & STAT5 \\
\hline & IL-3 & JAK2 & STAT5 \\
\hline & IL-4 & JAK1, JAK3 & STAT6 \\
\hline & IL-5 & JAK2 & STAT5 \\
\hline & IL-6 & JAK1, JAK2, TYK2 & STAT1, STAT3, STAT5 \\
\hline & IL-7 & JAK1, JAK3 & STAT5 \\
\hline & IL-9 & JAK1, JAK3 & STAT3, STAT5 \\
\hline & IL-11 & JAK1, JAK2, TYK2 & STAT1, STAT3 \\
\hline & IL-12 & JAK2, TYK2 & STAT4 \\
\hline & IL-13 & JAK1, JAK2, TYK2 & STAT6 \\
\hline & IL-15 & JAK1, JAK3 & STAT3, STAT5 \\
\hline & IL-21 & JAK1, JAK2, TYK2 & STAT3, STAT5 \\
\hline & IL-23 & JAK2, TYK2 & STAT3, STAT4, STAT5 \\
\hline & IL-27 & JАK2, ТYК2 & STAT3, STAT4, STAT5 \\
\hline & IL-35 & JАK2, ТYK2 & STAT3, STAT4, STAT5 \\
\hline & EPO & JAK2 & STAT5 \\
\hline & TPO & JAK2 & STAT5 \\
\hline & $\mathrm{GH}$ & JAK2 & STAT5 \\
\hline & G-CSF & JAK2 & STAT3 \\
\hline & GM-CSF & JAK1, JAK2 & STAT5 \\
\hline & TSLP & JAK1, JAK2 & STAT3 \\
\hline
\end{tabular}




\begin{tabular}{|l|c|c|c|}
\hline \multirow{2}{*}{ Type Il cytokine receptors } & INF- & JAK1, TYK2 & STAT1 \\
\cline { 2 - 3 } & INF- $\alpha / \beta$ & JAK1, TYK2 & STAT1, STAT2, STAT4 \\
\hline & IL-10 & JAK1, JAK2, TYK2 & STAT1, STAT3, STAT5 \\
\hline & IL-19 & JAK1, JAK2, TYK2 & STAT3 \\
\hline & IL-20 & JAK1, JAK2, TYK2 & STAT3 \\
\hline & IL-22 & JAK1, JAK2, TYK2 & STAT1, STAT3, STAT5 \\
\hline IL-24 & JAK1, JAK2, TYK2 & STAT1 \\
\hline & IL-26 & JAK1, JAK2, TYK2 \\
\hline
\end{tabular}

EPO, Erythropoietin; G-CSF, Granulocyte Colony-Stimulating Factor; GH, Growth Hormone; GM-CSF, Granulocyte-Macrophage Colony-Stimulating Factor; IFN, Interferon; IL, Interleukin; TPO, Thrombopoietin; TSLP, Thymic Stromal Lymphopoietin.

suppressing further signaling events [6,11,14,17]. Another regulatory module involves proteins of the PIAS ('Protein Inhibitor of Activated STAT') family which suppress STAT-driven gene transcription $[6,18]$.

\section{The JAK inhibitors (JAKi)}

Many cytokines that use the JAK-STAT signaling pathway are critical regulators of immune-mediated diseases. Therefore, pharmacological inhibition of this signal transduction pathway through JAKi may benefit a variety of inflammatory and autoimmune disorders [3,5,6,9-12,15].

Differently than biologics, which target cytokines by intravenous or subcutaneous injection, JAKi target cytokine signaling by either oral or topical administration. Besides,JAKi have short half-lives $[3,5,9,11,12,15]$. As activation of JAK-STAT pathway is through recruitment and phosphorylation of a pair of JAKs, inhibition of one of the JAKs enzymes is able to suppress the whole JAK-STAT signaling pathway[3].

The four JAKs have a very similar chemical structure with seven distinct homologous regions which can be divided into four structural domains (Figure 2) [1,3,6,10,15]:

- $\quad$ FERM-domain (JH5, JH6, JH7): interacts directly with the intracellular domain of the cytokine receptor.

- $\quad \mathrm{SH} 2$ domain $(\mathrm{JH} 3, \mathrm{JH} 4)$ : also interacts directly with the intracellular domain of the cytokine receptor and is supposed to play a structural role.

- $\quad$ Pseudo kinase domain (JH2): not functional catalytically and may have a regulatory role; the region most affected by oncogenic mutations

- Kinase domain (JH1): where the Adenosine Triphosphate (ATP) binds.

JAKi act by competitively blocking the ATP binding sites in the JH1 domains through non-covalent interactions (Figure 2) $[1,3,14,15]$. Because the JH1 domains of the four JAKs exhibit a high degree of homology, the development of selective JAKi has been challenging even under the direction of JAKs crystal structures $[1,3,9,10,14,15]$. Until now, it has not been possible to design a JAKi that specifically inhibits only one JAK $[1,9,10,14,15]$.
First-generation or classic JAKi (e.g. tofacitinib, ruxolitinib, baricitinib) selectively block two or more JAKs $[4,9,11,15]$ and can affect the patient's hematopoiesis and immune status [3,9$11,15]$. In general, the studies have shown an acceptable safety profile, with infection and cytopenia being the major adverse events of the JAKi $[3,9,10]$.

In relation to infections, increased risk of minor infections (nasopharyngitis, gastroenteritis, viral upper respiratory tract infections) as well as some serious infections (e.g. tuberculosis, cellulitis, panniculitis) is observed in patients treated with JAKi [9-11,15,17]. The risk of infection has been shown to be similar to that observed with disease-modifying antirheumatic drugs (DMARDs) and biologics, but the risk of reactivation of varicella zoster virus seems to be greater with JAKi[10,11,15]. The risk of this complication is increased by the associated use of JAKi with steroids and MTX [11] so, previous vaccination is recommended $[4,11]$. Considering all the cytokines that are blocked by inhibiting JAKs, it is quite surprising that the rate of infections is not greater than the observed with other immunosuppressors $[10,11,15]$. One explanation for this may be that many cytokines like TNF, IL1, IL-8, and IL-17 are not dependent upon JAKs [1,11,14].

Other JAKi side effects include anemia, leukopenia, thrombocytopenia, hyperlipidemia (increase in LDL and HDL cholesterol values as well as triglycerides), headache, nausea, diarrhea, increased creatine phosphokinase level (without causing muscle disease), and sporadic elevations of transaminases and creatinine $[4,9,11,15]$. It is worth mentioning that venous thrombosis, lymphoma, and other malignancies are rare adverse events reported in patients treated with JAKi $[4,11]$. No increased risk for malignancy has been reported in patients with rheumatoid arthritis treated with JAKi. Accumulation of cases with these rare serious adverse events are needed to evaluate their real risks [17].

Classics JAKi target multiple cytokines simultaneously which is not necessarily a disadvantage regarding efficacy, but of course, may bear a higher risk for toxicities. On the other hand, the second-generation or new generation JAKi possess much larger affinity for one JAK enzyme $[1,3,9,11,15]$. So, selective JAKi have the potential to limit the activity of a smaller subset of ligands and thereby enable signaling via other JAK-dependent pathways to be 


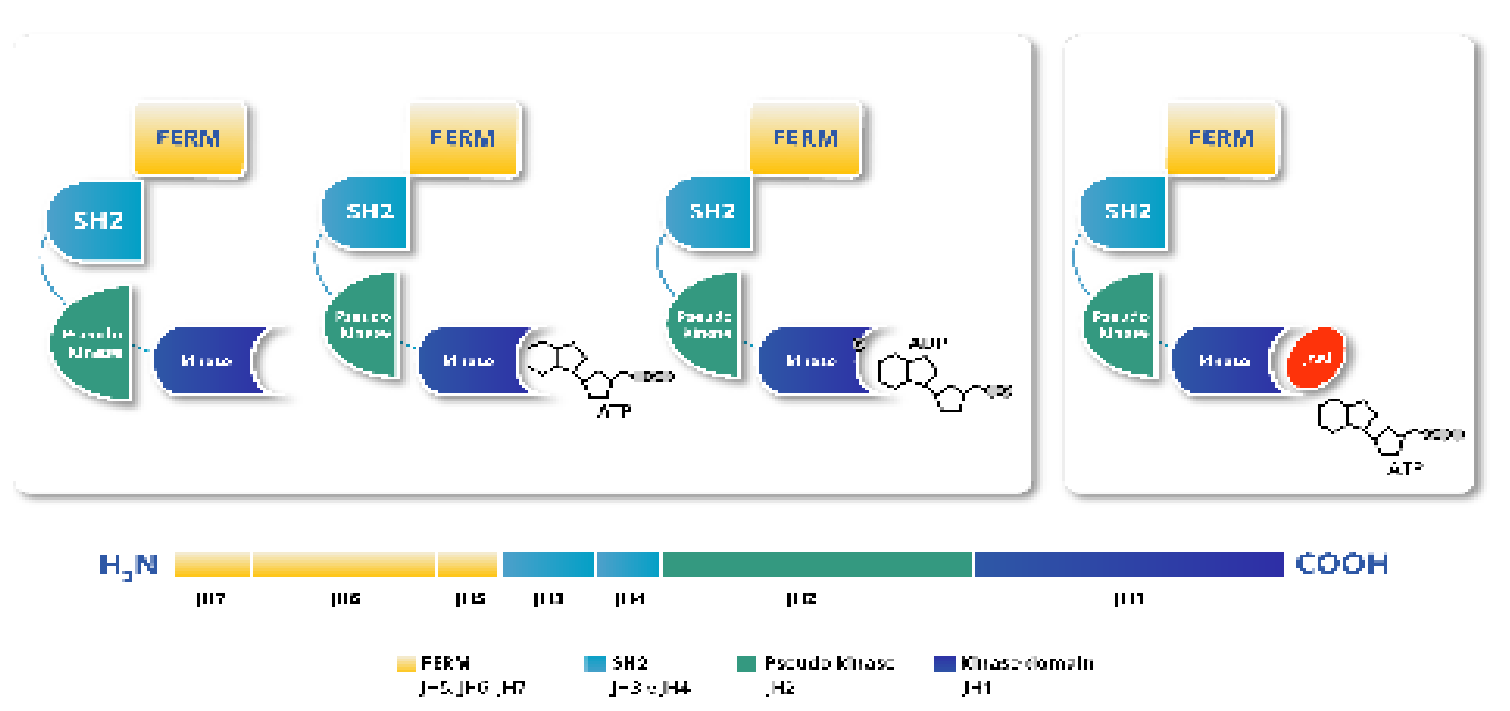

Figure 2: Upon the cytokine binding to its receptors, JAKs acts by transferring y-phosphate from Adenosine Triphosphate (ATP) to tyrosine residues on other proteins, including JAKs themselves, cytokine receptors, and the STATs, leading to STATs dimerization and subsequent nuclear translocation for gene regulation. The four JAKs have a very similar chemical structure with seven distinct homologous regions which can be divided into four structural domains: FERM-domain (JH5, JH6, JH7), SH2 domain (JH3, JH4), Pseudo kinase domain (JH2), and Kinase domain (JH1). JAKi are ATPcompetitive target the JH1 tyrosine kinase domain and block cytokine signaling by inhibiting kinase activity.

maintained which, potentially, reduce the incidence of adverse effects $[1,3,4,9,10,11,16]$. For example, JAK1 can pair with the other three JAKs and the JAK1-selective inhibitors target the broadest cytokine profile among JAKs. However, selective JAK1 inhibitors still spare JAK2-dependent Erythropoietin (EPO) and Thrombopoietin (TPO) pathways responsible for adverse effects arising from JAK2 inhibition such as anemia, neutropenia, and thrombocytopenia $[3,9,10,11]$. Thus, JAK1-selective inhibitors have a more favorable hematologic adverse effect profile than a pan-JAKi [3,9-11]. However, hematologic abnormalities are still reported with JAK1 inhibitors, possibly due to the inhibition of IL-6 signaling or by a residual presence of a JAK2 inhibitory effect $[9,11]$.

In summary, JAKi can have overlapping but distinct mechanisms of action, efficacy, and safety profiles $[4,9,11,15]$. The selectivity of the JAKi, as well as the doses employed, the intrinsic characteristics of the diseases and of the patients under treatment are factors related to the overall safety profile[4,9,11]. For instance, in AD patients, adverse events reported with oral JAK1-selective inhibitors in clinical trials have been mild, including headache, nausea, minor infections, and acne. This more favorable safety profile can be partially attributed to a younger, healthier patient cohort in $\mathrm{AD}$ [9].

\section{Conclusion}

Advanced understanding of the JAK-STAT pathway has led to the development of the first generation JAKi (which can block more than one JAK) and nowadays of selective JAKi (which have more affinity for one JAK, yet being able to block others JAKs, especially at higher doses) $[1,3,4,9,12]$. JAKi effectively treat a multitude of hematologic and inflammatory diseases [1,4,6,12,15]. Currently, multiple oral and topical JAKi are being investigated for many inflammatory dermatoses $[4,9,11,12]$, emerging as new and promising treatments.

In rheumatology, approved oral JAKi regimens are currently based on single doses both for induction and remission $[3,5,9,11,15]$. On the other hand, the possibility of dose optimization is very interesting and probably will be employed in the future alternative regimes. For instance, alternative regimens could include starting with a higher dose to induce remission and thenceforth maintain a lower dose or starting with the higher dose and reducing it just after remission is achieved.

In conclusion, due to the large number of clinical trials that are currently being carried out in several skin diseases $[5,9,12]$, dermatologists should understand the mechanism of action of JAKi as well as wait for the ongoing clinical trials results which will decide about the real-world efficacy and safety profiles of this new encouraging class of immunomodulators.

\section{Declarations}

Ana Paula Galli Sanchez has served as a speaker and/or consultant to AbbVie, Janssen, Lilly, Novartis, Pfizer, Sanofi, and Sandoz.

Tatiane Ester Aidar Fernandes and Gustavo Martelli Palomino were Abbvie's employees at the time of article writing. 


\section{References}

1. Clark JD, Flanagan ME, Telliez JB. Discovery and development of Janus kinase (JAK) inhibitors for inflammatory diseases. J Med Chem. 2014;57(12):5023-5038. doi: 10.1021/jm401490p.

2. Schwartz DM, Bonelli M, Gadina M, O'Shea JJ. Type I/II cytokines, JAKs, and new strategies for treating autoimmune diseases. Nat Rev Rheumatol. 2016;12(1):25-36. doi: 10.1038/nrrheum.2015.167.

3. Xu P, Shen P, Yu B, Xu X, Raoling G, Cheng X, et al. Janus kinases (JAKs): The efficient therapeutic targets for autoimmune diseases and myeloproliferative disorders. Eur J Med Chem. 2020;192:112155. doi: 10.1016/j.ejmech.2020.112155.

4. Gadina M, Chisolm DA, Philips RL, McInness IB, Changelian PS, O’Shea JJ. Translating JAKs to Jakinibs.J Immunol. 2020;204(8):2011-2020. doi 10.4049/jimmunol.1901477.

5. Montilla AM, García F, Arias PJ, Jesus GM, Jorge HP, et al. Scoping Review on the Use of Drugs Targeting JAK/STAT Pathway in Atopic Dermatitis, Vitiligo, and Alopecia Areata. DermatolTher (Heidelb). 2019;9(4):655683. doi: 10.1007/s13555-019-00329-y.

6. Villarino AV, Kanno Y, Shea JJ. Mechanisms and consequences of JakSTAT signaling in the immune system. Nat Immunol. 2017;18(4):374384. doi: 10.1038/ni.3691.

7. Damsky W, King BA. JAK inhibitors in dermatology: The promise of a new drug class. J Am AcadDermatol. 2017;76(4):736-744. doi: 10.1016/j.jaad.2016.12.005.

8. Hassidim SR, Ramot Y, Zlotogorski A. Janus kinase inhibitors in dermatology: A systematic review. J Am AcadDermatol. 2017;76(4):745-753. doi: 10.1016/j.jaad.2016.12.004.

9. He H, Yassky E. JAK Inhibitors for Atopic Dermatitis: An Update. Am J ClinDermatol. 2019;20(2):181-192. doi: 10.1007/s40257-018-0413-

10. Virtanen AT, Haikarainen T, Raivola J, Silvennoinen O. Selective
JAKinibs: Prospects in Inflammatory and Autoimmune Diseases. BioDrugs. 2019;33(1):15-32. doi: 10.1007/s40259-019-00333-w.

11. Gadina M, Le MT, Schwartz DM, Silvennoinen O, Nakayamada S, et al. Janus kinases to jakinibs: from basic insights to clinical practice. Rheumatology (Oxford). 2019;58(Suppl1):i4-i16. doi: 10.1093/ rheumatology/key432.

12. Solimani F, Meier K, Ghoreschi K. Emerging Topical and Systemic JAK Inhibitors in Dermatology. Front Immunol. 2019;10:2847. doi: 10.3389/fimmu.2019.02847.

13. Turner MJ. Cytokines and chemokines. In: Anthony A. Gaspari, Sthephen K. Tyring, Daniel H. Kaplan, editors. Clinical and Basic Immunodermatology. $2^{\text {nd edition, Springer:2017;pp:239-264. }}$

14. Morris R, Kershaw NJ, Babon JJ. The molecular details of cytokine signaling via the JAK/STAT pathway. Protein Sci. 2018;27(12):19842009. doi: $10.1002 /$ pro.3519.

15. Matencio JM, Baladrón MA, Castaneda S. JAK-STAT inhibitors for the treatment of immunomediated diseases.Med Clin (Barc). 2019;152(9):353-360. doi: 10.1016/j.medcli.2018.10.020.

16. Leonard WJ, Lin JX, O'Shea JJ. The $\gamma c$ Family of Cytokines: Basic Biology to Therapeutic Ramifications. Immunity. 2019; 50(4): 832-850. doi: 10.1016/j.immuni.2019.03.028.

17. Harigai M. Growing evidence of the safety of JAK inhibitors in patients with rheumatoid arthritis.Rheumatology (Oxford). 2019;58(Suppl1):i34-i42. doi: 10.1093/rheumatology/key287.

18. Niu GJ, Xu JD, Yuan WJ, Sun J, Yang MC, et al. Protein Inhibitor of Activated STAT (PIAS) Negatively Regulates the JAK/STAT Pathway by Inhibiting STAT Phosphorylation and Translocation. Front Immunol. 2018;9:2392. doi: 10.3389/fimmu.2018.02392.

19. Yoshimura A, Suzuki M, Sakaguchi R, Hanada T, Yasukawa H. SOCS, Inflammation, and Autoimmunity. Front Immunol. 2012;3:20. doi: 10.3389/fimmu.2012.00020. 